\title{
Neurocalcin immunoreactivity in the rat accessory olfactory bulb
}

Angel Porteros ${ }^{1}$, Jesús G. Briñón ${ }^{1}$, Carlos Crespo ${ }^{1}$, Katsuo Okazaki², Hirayoshi Hidaka², José Aijón ${ }^{1}$ and José R. Alonso ${ }^{1}$

Institutional affiliation:

1.- Departamento de Biología Celular y Patología

Universidad de Salamanca

E-37007 Salamanca

Spain

2.- Department of Pharmacology

Nagoya University School of Medicine

Nagoya 466

Japan

Running title: Neurocalcin in the rat AOB

Correspondence to be sent to:

Dr. J.R. Alonso

Departamento de Biología Celular y Patología

Universidad de Salamanca

E-37007 Salamanca

Spain

Phone number: +34-23-294400 ext. 1854

Fax number: +34-23-294549

e-mail: jralonso@gugu.usal.es 


\section{SUMMARY}

The distribution and morphology of neurocalcin-immunopositive neurons have been studied in the rat accessory olfactory bulb. Different subsets of neurons displaying neurocalcin-immunoreactivity were found in the glomerular layer, the external plexiform layer and the internal plexiform layer. The most abundant staining was detected in the glomerular layer where neurocalcin-immunoreactive periglomerular cells and external tufted cells were observed in the lateral glomeruli, whereas the central region of this layer was practically devoid of immunopositive neurons. In the external plexiform layer, medial tufted cells and Van Gehuchten cells displayed neurocalcin-immunoreactivity. In the internal plexiform layer, interneurons classified as horizontal cells and vertical cells of Cajal were neurocalcin-immunostained. The staining pattern for neurocalcin in the accessory olfactory bulb showed similarities with the immunostaining described in this brain region for another EF-hand calcium binding protein, calbindin D-28k. However, after double immunohistochemical labeling, colocalization of both proteins in the same neuron was not observed, reflecting a biochemical heterogeneity within morphologically homogeneous neuronal groups.

\section{KEY WORDS}

Accessory olfactory system, calcium-binding protein, immunohistochemistry, neurocalcin, rat, vomeronasal organ. 


\section{INTRODUCTION}

Neurocalcin (NC) is a recently described calcium-binding protein belonging to the EF-hand family which possess three $\mathrm{Ca}^{2+}$-binding domains $17,19,26$. NC shows extensive homology with visinin and recoverin, also members of the three EF-hand group, but not with the first described protein of this family, parvalbumin ${ }^{19}$. The amino acid sequence of the calcium-binding domains is more closely related to that of calmodulin ${ }^{18}$. All these proteins are considered to modulate or to mediate the action of free intracellular calcium in neurons ${ }^{3}$. In the case of NC, a mechanism of molecular actuation similar to that observed for myristoylated recoverin in the optic receptors has been suggested 4,12 , since NC has the consensus amino-terminal sequence for myristoylation. This sequence seems important for the membrane linking of the protein in a calcium-dependent way 29 and its interaction with guanylate cyclase ${ }^{10}$.

The accessory olfactory bulb (AOB) is the first relay station for the axons coming from the vomeronasal organ to the brain. Axons from the vomeronasal receptor cells arrive to the AOB where they form the vomeronasal nerve layer (VL). These axons contact with dendrites of mitral cells, tufted cells, and periglomerular cells in the glomerular layer (GL). In the AOB, mitral cells do not form a single row arrangement, but they are scattered in the external plexiform layer (EPL). The internal plexiform layer (IPL) is wide, and clearly distinguishable since it is occupied by the myelinated fibers of the lateral olfactory tract, formed by the axons of output neurons located in the MOB and AOB. Numerous dendrites of granule cells located in the innermost stratum, the granule cell layer (GCL), cross the IPL in dense bundles directed towards the EPL. The main neuronal circuit within the AOB is formed by the primary synaptic contact between the axons from the vomeronasal receptor cells with dendrites of output neurons (mitral and tufted cells), which send 
their axons to higher brain areas $9,24,25$. This monosynaptic pathway is modulated by different classes of intrinsic interneurons of the AOB. In the rat, the AOB is thought to control olfactory recognition between conspecifics ${ }^{7}$ and it is involved in reproductive and maternal behavior $6,11,28$.

Previous studies reported the presence of NC-immunoreactivity in the main olfactory bulb (MOB) ${ }^{4}$, olfactory epithelium and vomeronasal organ ${ }^{5,12}$, inner ear ${ }^{13}$, spinal cord and dorsal root ganglia 20 , retina ${ }^{17}$, and adrenal gland 18 . No comprehensive study is available on the distribution of NC in the AOB. The aim of the present study is to describe the NC containing neurons in the $\mathrm{AOB}$ and to investigate the possible coexpression of this protein with other calcium-binding proteins such as parvalbumin (PV), calretinin (CR) and calbindin D-28k (CB) whose distribution patterns in the olfactory bulb are already known 1,8,21-23.

\section{MATERIALS AND METHODS}

Five adult female Wistar rats weighing between 200 and 250 g were used. The animals were deeply anaesthetized with ketamine (Ketolar, $50 \mathrm{mg} / \mathrm{kg}$ body weight) and perfused intraaortically with $100 \mathrm{ml}$ saline solution followed by $400 \mathrm{ml}$ of a fixative made up of $4 \%$ paraformaldehyde and $15 \%$ saturated picric acid in $0.1 \mathrm{M}$ phosphate buffer, $\mathrm{pH} 7.3$ (PB). After being removed from the skull, the brains were dissected out and postfixed at $4^{\circ} \mathrm{C}$ for four hours in the same fixative solution. The blocks containing the AOB were cryoprotected in $30 \%$ sucrose in $\mathrm{PB}$ at $4^{\circ} \mathrm{C}$, and 30 $\mu \mathrm{m}$ thickness sections were cut on a Leica cryostat in both coronal and sagittal planes.

NC immunohistochemistry 
Free-floating sections were washed in PB and incubated for thirty minutes in $10 \%$ normal goat serum in PB. Thereafter, they were incubated in anti-NC primary antibody, raised in rabbit against rat brain $\mathrm{NC}^{17}$, diluted 1:8000 in $\mathrm{PB}$, for 48 hours at $4^{\circ} \mathrm{C}$. Then, the sections were processed according to the avidin-biotinimmunoperoxidase method. The sections were washed (3 x $10 \mathrm{~min})$ in PB and incubated for 1 hour at room temperature in biotinylated anti-rabbit immunogammaglobulin (Vectastain, Vector Laboratories, Burlingame, USA) diluted 1:250 in PB. After washing in PB (3 x $10 \mathrm{~min}$ ), sections were transferred to Vectastain ABC reagent diluted 1:200 in PB for 2 hours. The immunocytochemical reaction was visualized using $0.05 \%$ 3,3'-diaminobenzidine tetrahydrochloride (Sigma) with $0.003 \%$ hydrogen peroxide in $0.2 \mathrm{M}$ Tris-HCl buffer, $\mathrm{pH}$ 7.6. Sections were then rinsed in PB, mounted on gelatin-coated slides, air-dried, dehydrated through graded ethanol series, cleared in xylene and coverslipped with Entellan (Merck). The sections were examined in a Zeiss III microscope. Cell measures were made using a Zeiss ocular micrometer.

\section{Analysis of coexistence of NC with $C B$}

The analysis of the coexistence of NC with $\mathrm{CB}$ in the same neuron was carried out using a double immunofluorescence technique. After preincubation, $30 \mu \mathrm{m}$ cryostat sections were incubated for 48 hours at $4^{\circ} \mathrm{C}$ in a mixture of rabbit anti-NC diluted 1:4000 and mouse anti-CB diluted 1:1000 in PB. After several washes in PB, the sections were incubated for 2 hours at room temperature in a mixture of horse anti-mouse FITC-conjugated immunogammaglobulin and goat anti-rabbit Texas red conjugated immunogammaglobulin, diluted 1:75 in PB. After washing in PB, sections were mounted on gelatin-coated slides, and coverslipped with an anti-fading medium (0.42 g glycine, $0.021 \mathrm{~g}$ sodium hydroxide, $0.51 \mathrm{~g}$ sodium chloride, $0.03 \mathrm{~g}$ sodium azide, and 5 g N-propyl gallate in $100 \mathrm{ml} \mathrm{70 \%} \mathrm{glycerin).} \mathrm{Labeled} \mathrm{cells} \mathrm{were}$ 
identified in a Zeiss III photomicroscope equipped with epifluorescence and appropriate filter sets.

\section{Controls}

The primary anti-NC antibody used have been fully characterized by immunoblotting ${ }^{17}$ and does not cross-react with other brain proteins. The specificity of the NC antibody has been tested by pre-absorbtion with purified NC from bovine brain $4,12,13,17,20$. Controls of the immunocytochemical procedure by omitting the primary antibody were also carried out. Additionally, interference by endogenous peroxidases was ruled out by staining some sections with chromogen and hydrogen peroxide. Residual staining was not observed in any case throughout the AOB.

\section{RESULTS}

After NC-immunohistochemistry, immunopositive neurons displayed a Golgilike staining, enabling a good morphological characterization of positive neurons, with densely stained cell bodies, dendrites and in some occasions the initial portions of the axons. NC-immunoreactive neurons were observed in the GL, EPL and IPL. Table I shows the NC-immunoreactive cell types, their somatic sizes and their frequency. Figure 1 shows schematically the distribution of NC-immunopositive neurons in the rat AOB. In order to allow a better comparison, the distribution of NC-labeling in the MOB is showed in the same figure.

Vomeronasal fibers and all glomeruli in which they arborize were completely negative. The most prominent staining was obtained in the GL, where a segregated topographical distribution of NC-immunopositive cells was observed. Numerous NC-immunoreactive neurons surrounded the glomeruli located in both the lateral and medial sides of the AOB, but only scattered NC-labeled neurons were observed 
in the central glomeruli (Fig. 2a). Two different populations of NC-immunopositive cells were observed in this layer. The first population was formed by neurons with 10-15 $\mu \mathrm{m}$ of maximum diameter, with spherical or fusiform somata, and strong immunostaining intensity. Most of these cells were located at the boundary with the EPL. They showed one or two aspiny dendrites. Frequently, the dendrites were observed entering and branching profusely inside a single glomerulus (Fig. 2b, c). The initial segments of the axons of these cells were occasionally observed oriented towards the inner regions of the AOB. According to these observations, these neurons were typified as external tufted cells.

The other NC-immunoreactive neuronal population observed in the GL was formed by smaller neurons, with ovoid or round cell bodies, ranging 8-10 $\mu \mathrm{m}$ in size. These cells were also located around the glomeruli, and with the same segregated distribution at both lateral sides of the GL. They showed one dendritic trunk which innervated a single glomerulus. The axons of these cells were not clearly observed in our preparations. The neurons belonging to this second NCimmunostained population of the GL were identified as periglomerular neurons (Fig. 2e).

In the internal region of the EPL, a population of scattered NC-immunoreactive neurons corresponding to the middle tufted cell type was observed. The cell bodies were fusiform with major diameters ranging from 11 to $14 \mu \mathrm{m}$. They showed a thick and smooth dendrite oriented towards the EPL that reached the GL and entered a single glomerulus (Fig. 2d). In some cells, the initial portion of the axon was observed.

Another type of NC-immunopositive neuron of the EPL was formed by cells showing round of ovoid somata with sizes between 10 and $15 \mu \mathrm{m}$ in maximum 
diameter. The cell body gave rise from opposite poles to a variable number of varicose dendrites that branched close to it (Fig. 2f). Axon-like prolongations were not observed. These cells resembled the morphological characteristics of Van Gehuchten cells.

Two different populations were NC-immunopositive in the IPL. One NCimmunopositive neuronal type observed in the IPL was identified as vertical cells of Cajal. They possessed spherical, or ovoid neuronal somata with maximum diameters ranging between 10 and $13 \mu \mathrm{m}$. These neurons showed two main dendrites emerging from opposite poles of the somata, oriented perpendicular to the lateral olfactory tract fibers coursing in the IPL (Fig. 3a, b). The other population was constituted by horizontal cells, with round or oval somata (12-15 $\mu$ m maximum diameter). These neurons had two dendrites arising from opposite poles of the somata and coursing parallel to the AOB lamination (Fig. 3c, d).

The distribution of NC-positive neurons in the AOB showed important differences with that of CR since vomeronasal axons and a large population of granule cells were CR-immunopositive. Therefore, comparing the distribution of CR-immunopositive neurons with that described for NC, coexistence of both proteins in the same cell was unexpected. By contrast, comparing the distribution of NC and CB, there were subpopulations of the same neuronal types displaying both immunoreactivities. Thus, particular subpopulations of periglomerular cells, horizontal cells and vertical cells of Cajal displayed NC immunoreactivity and CB immunoreactivity. However, after a detailed examination of double-stained sections for these proteins, no double-labeled cell was detected reflecting the presence of these proteins in entirely separate neuronal subpopulations (Fig. 4).

\section{DISCUSSION}


The present study demonstrates that NC is expressed by a large variety of neuronal populations in the rat AOB: projecting neurons (external and middle tufted cells) and interneurons (periglomerular cells, Van Gehuchten cells, horizontal cells and vertical cells of Cajal). These data contrast with those reported for $\mathrm{CB}^{21}$ that is restricted to specific populations of interneurons. Nevertheless, since all mitral cells and internal tufted cells and most middle tufted cells were NC-immunonegative, the bulk of the projection arising in the AOB did not express this calcium-binding protein.

Iino and coworkers ${ }^{12}$ have described the presence of NC-immunoreactivity in most, if not all, mature vomeronasal receptors, and in the vomeronasal axon bundles crossing the lamina propia, suggesting and important role for this protein in odorant signal transduction. By contrast, we did not observe NC-immunolabeling in the vomeronasal axons either in the VL or in the GL. Similar results were obtained in the main olfactory system where a large number of olfactory receptors were NCimmunoreactive $^{5}$ and, however, the axons arising from these cells and the olfactory nerve layer and glomerular layer of the main olfactory bulb lacked NCimmunoreactivity 4 , 5. This discrepancy may be due to the absence of axonic transport of this protein or that the amount of NC in the axon terminals was too low to be detected by immunohistochemistry.

NC was detected in a spatially segregated subpopulation of external tufted cells, specifically located at the lateral and medial glomeruli, but not in the central ones. NC immunoreactivity has been described in external tufted cells of the $\mathrm{MOB}^{4}$ but they were homogeneously distributed in all glomeruli. These findings indicate that NC could be involved in the intrabulbar modulation of particular groups of signals in the accessory olfactory system. In this sense, specific subsets of vomeronasal axons 
project to specific regions of the glomerular layer in the $\mathrm{AOB}^{15}$. Thus, the asymmetric distribution of NC-immunoreactive external tufted cells in the GL may be related to a specific connectivity pattern of vomeronasal axons to certain output neurons, as described with specific odor stimulation in the mitral cells of the MOB 16

Comparing the distribution of $\mathrm{NC}$ in the $\mathrm{AOB}$ with previous reports on the immunocytochemical localization of other calcium-binding proteins in the vomeronasal system $8,21-23$ both similarities and differences can be detected. PV immunoreactivity is completely absent in both the vomeronasal fibers and the intrinsic neurons of the $\mathrm{AOB}^{8}$. $\mathrm{CR}$ has been found in the vomeronasal axons and in a high number of granule cells 22,23 , which are NC-immunonegative. Data about the presence of CR in projection neurons (mitral and tufted cells) of the olfactory bulb are controversial. Some authors indicate the absence 23 of CR immunoreactivity, or only weak and doubtful staining14,22 that dissapears from mitral cells when the antiserum is preincubated with brain $\mathrm{CR}^{22}$. Combining $\mathrm{CR}$ immunocytochemistry with neuranatomical tracing and unilateral lesion of the olfactory bulb, it has been reported the presence of weak CR immunoreactivity in mitral cells but no immunoreactivity at all in tufted cells of the $\mathrm{MOB}^{27}$. By contrast, $\mathrm{NC}$ immunoreactivity has been detected in tufted cells of the $\mathrm{MOB}^{4}$ and in the $\mathrm{AOB}$ (present paper), whereas mitral cells were NC-immunonegative in the $\mathrm{MOB}^{4}$ and in the AOB (present paper).

Particular subpopulations of some neuronal types including periglomerular cells, horizontal cells and vertical cells of Cajal have been already described as CBimmunopositive 21 , and subpopulations of these same neuronal types have been found displaying NC-immunoreactivity. From our results, these NC- and CBimmunopositive cells formed entirely separate populations despite they correspond 
to the same morphological types. These chemical singularities in some neuronal types could reflect differences in their connectivity ${ }^{2}$, suggesting therefore the existence of different functional groups within these morphologically homogeneous cell types of the AOB.

\section{LIST OF ABBREVIATIONS}

AOB: Accessory olfactory bulb.

CB: Calbindin D-28k.

CR: Calretinin

DSA: Deep short-axon cell.

EPL: External plexiform layer.

ET: External tufted cell

GCL: Granule cell layer.

GL: Glomerular layer.

HZ: Horizontal cell.

IPL: Internal plexiform layer.

MCL: Mitral cell layer.

MOB: Main olfactory bulb.

MT: Middle tufted cell.

NC: Neurocalcin.

PG: Periglomerular cell.

PV: Parvalbumin

VC: Vertical cell of Cajal.

VG: Van Gehuchten cell.

VL: Vomeronasal nerve layer

WM: White matter. 
Porteros et al.

\section{ACKNOWLEDGMENTS}

This work was supported by grants from the DGICyT (PB94-1388 and PR95350) and the Junta de Castilla y León. 


\section{FIGURE LEGENDS}

\section{Figure 1}

Schematic drawing showing the NC-immunostaining patterns in the rat MOB and AOB. DSA: Deep short-axon cell. ET: External tufted cell. HZ: Horizontal cell. MT: Middle tufted cell. PG: Periglomerular cell. VC: Vertical cell of Cajal. VG: Van Gehuchten cell.

\section{Figure 2}

NC immunostaining in the superficial layers of the AOB. Scale bar= $150 \mu \mathrm{m}$ for a, $100 \mu \mathrm{m}$ for b, $50 \mu \mathrm{m}$ for c-f.

a.- Overview of the rat $\mathrm{AOB}$ showing NC-immunoreactive neurons in the glomerular layer. Note the abundance of NC-positive neurons in both lateral sides of the GL (solid arrows), whereas only scattered neurons were observed in the central region of the GL (open arrow).

b.- Glomerulus (G) surrounded by dense NC-immunostaining. Note the presence of intensely stained external tufted cells (arrow).

c.- Higher magnification showing the same cell indicated with an arrow in (b)

d.- NC-immunoreactive middle tufted cell (arrow) with an apical dendrite entering one glomerulus.

e.- NC-immunopositive periglomerular cells (arrows).

f.- NC-labeled Van Gehuchten cell located in the EPL. Two dendritic trunks (open arrows) arise from opposite poles of the cell body (arrow).

\section{Figure 3}

NC immunostaining in the deep layers of the rat AOB. Scale bar= $50 \mu \mathrm{m}$ (for all figures). 
a-b.- NC-immunostained vertical cells of Cajal located at the innermost region of the EPL (a), and at the IPL/GCL boundary (b) with an apical dendrite crossing through the IPL and directed towards the EPL (arrows).

c-d.- NC-immunopositive horizontal cells (arrows). (c) is a sagittal view and (d) is a coronal view of the IPL of the AOB.

\section{Figure 4}

Double immunofluorescence labeling with anti-NC (a,c) and anti-CB (b,d). Note the absence of colocalization (arrows) of both immunoreactivities in the GL $(a, b)$ and in the IPL (c,d). Scale bar= $50 \mu \mathrm{m}$ (for all figures). 


\section{REFERENCES}

1. Alonso, J.R., Arévalo, R., García-Ojeda, E., Porteros, A., Briñón, J.G. and Aijón, J., NADPH-diaphorase active and calbindin D-28k-immunoreactive neurons and fibers in the olfactory bulb of the hedgehog (Erinaceus europaeus), J. Comp. Neurol., 351 (1995) 307-327.

2. Andressen, C., Blümcke, I. and Celio, M.R., Calcium-binding proteins: selective markers of nerve cells, Cell Tissue Res., 271 (1993) 181-208.

3. Baimbridge, K.G., Celio, M.R. and Rogers, J.H., Calcium-binding proteins in the nervous system, Trends Neurosci., 15 (1992) 303-308.

4. Bastianelli, E., Okazaki, K., Hidaka, H. and Pochet, R., Neurocalcin immunoreactivity in rat olfactory bulb, Neurosci. Lett., 161 (1993) 165-168.

5. Bastianelli, E., Polans, A.S., Hidaka, H. and Pochet, R., Differential distribution of six calcium-binding proteins in the rat olfactory epithelium during postnatal development and adulthood, J. Comp. Neurol., 354 (1995) 395-409.

6. Bean, N.J. and Wysocki, C.J., Vomeronasal organ removal and female mouse aggression: The role of experience, Physiol. Behav., 45 (1989) 875-882.

7. Brennan, P., Kaba, H. and Keverne, B.J., Olfactory recognition: a simple memory system, Science, 250 (1990) 1223-1226.

8. Celio, M.R., Calbindin D-28k and parvalbumin in the rat nervous system, Neuroscience, 35 (1990) 375-475. 
9. De Olmos, J., Hardy, H. and Heimer, L., The afferent connections of the main and accessory olfactory bulb formations in the rat: An experimental HRP-study, $J$. Comp. Neurol., 181 (1978) 213-244.

10. Dizhoor, A.M., Ray, S., Kumar, S., Niemi, G., Spencer, M., Brolley, D., Walsh, K.A., Philipov, P.P., Hurley, J.B. and Stryer, L., Recoverin: a calcium sensitive activator of retinal rod guanylate cyclase, Science, 251 (1991) 915-918.

11. Gallego, A., Sánchez-Criado, J.E. and Mora, O., Role of the vomeronasal system in pheromonal reception in the rat. In J.E. Steimer and J.R. Ganchrow (Eds.), Determination of behaviour by chemical stimuli, IRL, London, 1981, pp. 57-64.

12. Iino, S., Kobayashi, S., Okazaki, K. and Hidaka, H., Neurocalcinimmunoreactive receptor cells in the rat olfactory epithelium and vomeronasal organ, Neurosci. Lett., (in press) (1995)

13. Iino, S., Kobayashi, S., Okazaki, K. and Hidaka, H., Immunohistochemical localization of neurocalcin in the rat inner ear, Brain Res., 680 (1995) 128-134.

14. Jacobowitz, D.M. and Winsky, L., Immunocytochemical localization of calretinin in the forebrain of the rat, J. Comp. Neurol., 304 (1991) 198-218.

15. Mori, K., Monoclonal antibodies (2C5 and 4C9) against lactoseries carbohydrates identify subsets of olfactory and vomeronasal receptor cells and their axons in the rabbit, Brain Res., 408 (1987) 215-221. 
16. Mori, K., Mataga, N. and Imamura, K., Differential specificities of single mitral cells in rabbit olfactory bulb for a homologous series of fatty acid odor molecules, J. Neurophysiol., 67 (1992) 786-789.

17. Nakano, A., Terasawa, M., Watanabe, M., Usuda, N., Morita, T. and Hidaka, H., Neurocalcin, a novel calcium binding protein with three EF-hand domains, expressed in retinal amacrine cells and ganglion cells, Biochem. Biophys. Res. Comm., 186 (1992) 1207-1211.

18. Nakano, A., Terasawa, M., Watanabe, M., Okazaki, K., Inoue, S., Kato, M., Nimura, Y., Usuda, N., Morita, T. and Hidaka, H., Distinct regional localization of neurocalcin, a $\mathrm{Ca}^{2+}$-binding protein, in the bovine adrenal gland., J. Endocrinol., 138 (1993) 283-290.

19. Okazaki, K., Watanabe, M., Ando, Y., Hagiwara, M., Terasawa, M. and Hidaka, H., Full sequence of neurocalcin, a novel calcium-binding protein abundant in central nervous system, Biochem. Biophys. Res. Comm., 185 (1992) 147-153.

20. Okazaki, K., Iino, S., Inoue, S., Kobayashi, S. and Hidaka, H., Differential distribution of neurocalcin isoforms in rat spinal cord, dorsal root ganglia and muscle spindle, Biochim. Biophys. Acta, 1223 (1994) 311-317.

21. Porteros, A., Arévalo, R., Crespo, C., García-Ojeda, E., Briñón, J.G., Aijón, J. and Alonso, J.R., Calbindin D-28k immunoreactivity in the rat accessory olfactory bulb, Brain Res., 689 (1995) 93-100.

22. Résibois, A. and Rogers, J.H., Calretinin in rat brain: an immunohistochemical study., Neuroscience, 46 (1992) 101-134. 
23. Rogers, J.H. and Résibois, A., Calretinin and calbindin D-28k in rat brain: patterns of partial co-localization, Neuroscience, 51 (1992) 843-865.

24. Scalia, F. and Winans, S.S., The differential projections of the olfactory bulb and accessory olfactory bulb in mammals, J. Comp. Neurol., 161 (1975) 31-56.

25. Smithson, K.G., Weiss, M.L. and Hatton, G.I., Supraoptic nucleus afferents from the accessory olfactory bulb: evidence from anterograde and retrograde tract tracing in the rat, Brain Res. Bull., 29 (1992) 209-220.

26. Terasawa, M., Nakano, A., Kobayashi, R. and Hidaka, H., Neurocalcin: a novel calcium-binding protein from bovine brain, J. Biol. Chem., 267 (1992) 1959619599.

27. Wouterlood, F.G. and Härtig, W., Calretinin-immunoreactivity in mitral cells of the rat olfactory bulb, Brain Res., 682 (1995) 93-100.

28. Wysocki, C.J., Neurobehavioral evidence for the involvement of the vomeronasal system in mammalian reproduction, Neurosci. Biobehav. Rev., 3 (1979) 301-341.

29. Zozulya, S. and Stryer, L., Calcium-myristoyl protein switch, Proc. Natl. Acad. Sci. USA, 89 (1992) 11569-11573. 

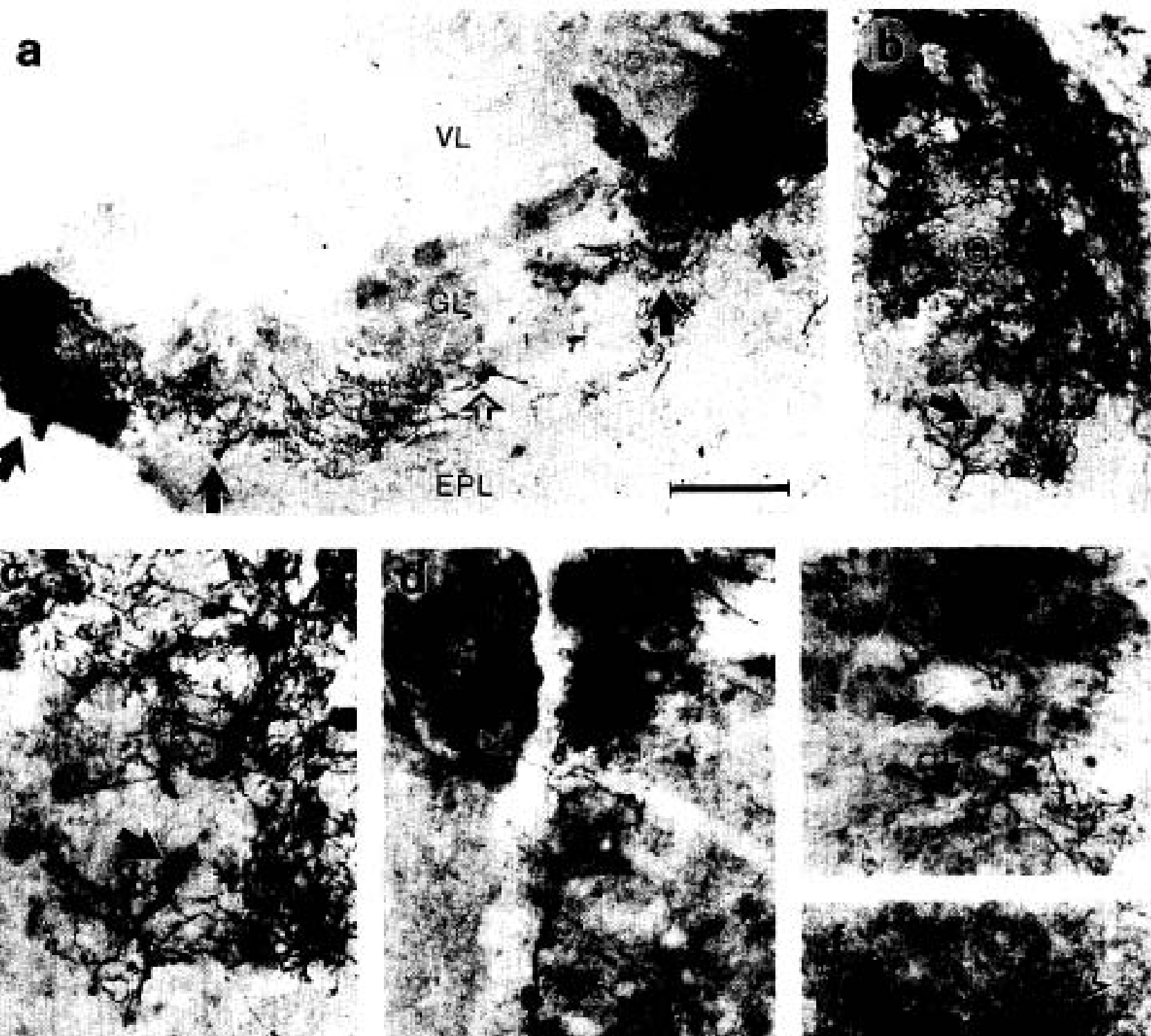

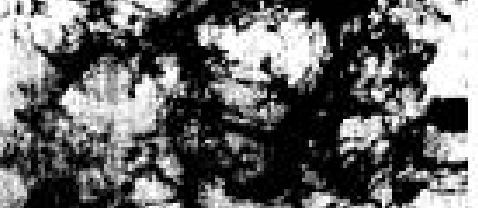

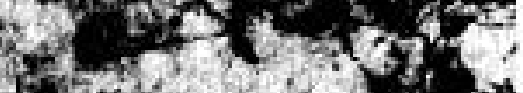

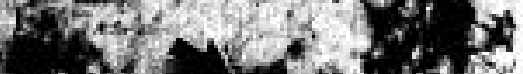

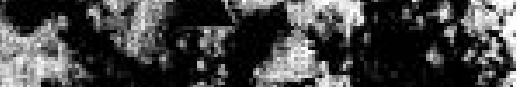
$x_{1} x^{2}+n^{2}$ is pros? $f$
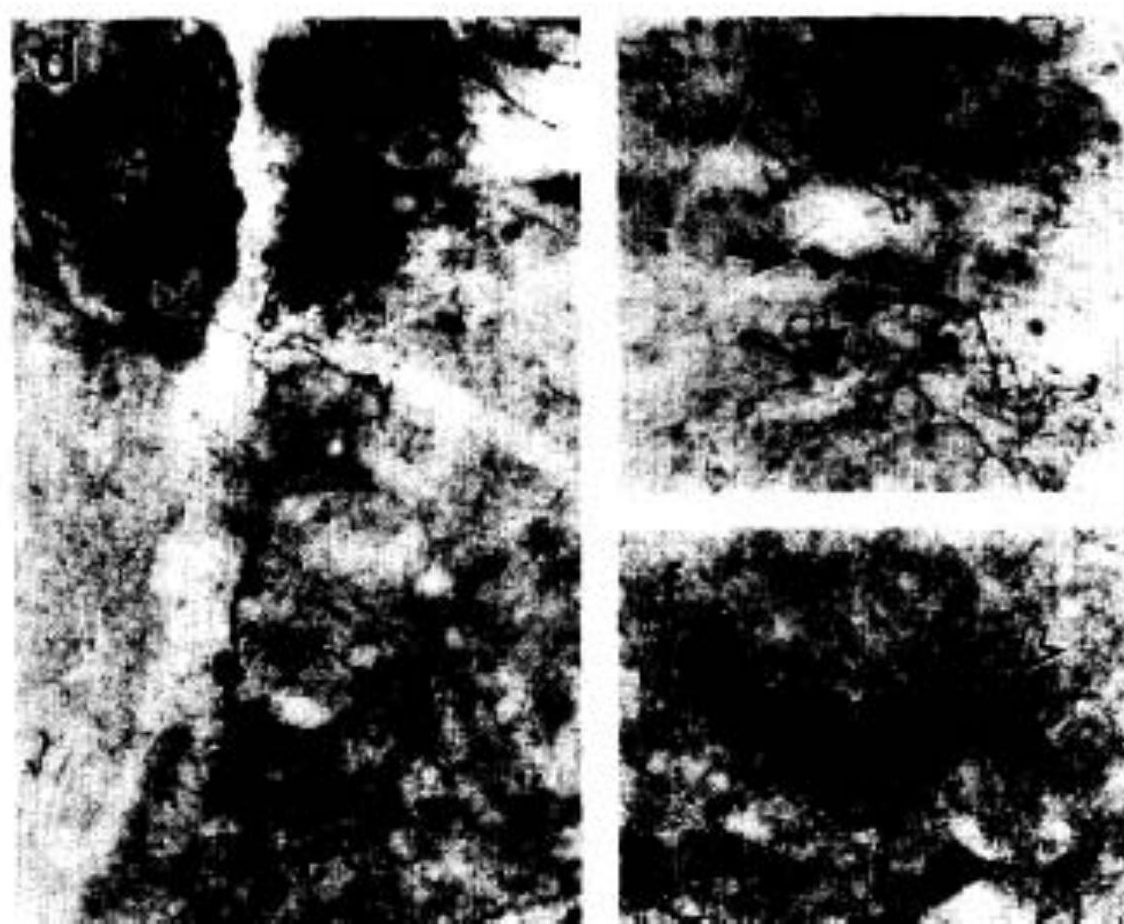


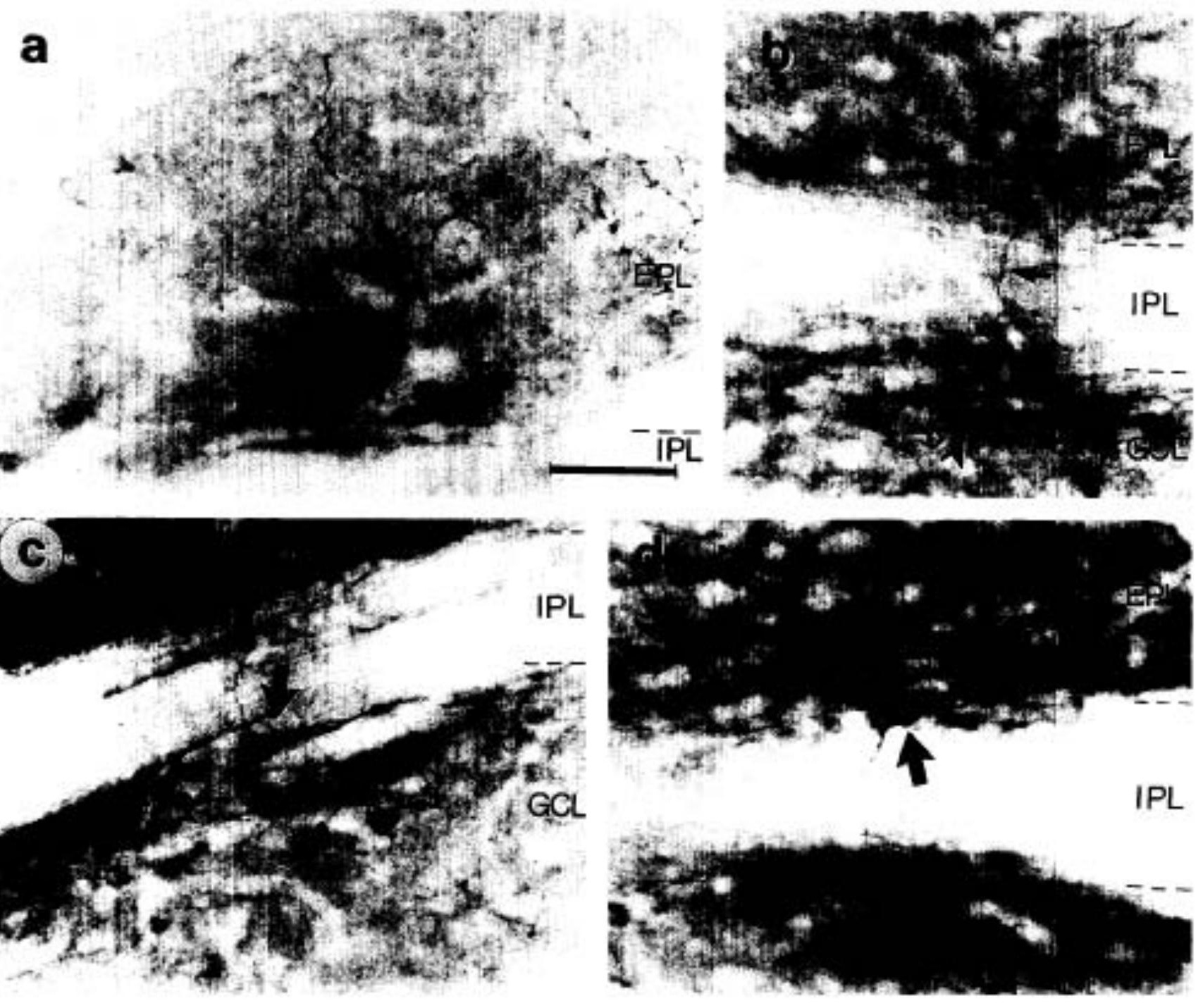


